\title{
THE SUN AT THE VLA'S METRIC AND DECIMETRIC WAVELENGTHS
}

\author{
S. M. White, M. R. Kundu, N. Gopalswamy and E. J. Schmahl \\ Astronomy Program \\ University of Maryland, College Park, MD 20742
}

During September 1988 (International Solar Month) we observed the Sun with the Very Large Array on 4 days in the period Sep. 11-17. The VLA was in its most compact configuration, which is ideal for studying large-scale coronal structures. Here we summarize some preliminary results of the observations at 0.333 and $1.5 \mathrm{GHz}$. Despite the presence of numerous active regions the sun was actually very quiet, with no flares during our observing, and this allowed us to make high-dynamicrange maps.

There is exceptionally good correspondence between the $1.5 \mathrm{GHz}$ radio sources and regions which are dark in the He10830 images and bright in the $\mathrm{Ca}$ images. This extends to very small weak features in the $1.5 \mathrm{GHz}$ radio maps, which nonetheless are found to have associated dark regions on the He images. Several filaments show up as depressions in active regions or as empty lanes. The $1.5 \mathrm{GHz}$ sources are generally very extended, appearing as a canopy straddling several nearby regions in one hemisphere. None of the active regions was particularly bright at 1.5 $\mathrm{GHz}$. The peak brightness temperatures of the active region sources on the four days are $1.7,1.6,1.5$ and $1.8 \times 10^{6}$ on Sep. $11,12,13$ and 17 respectively. However, on all days the range of peak brightness temperatures in different active regions was remarkably small. Typically there were about eight active region peaks present, each in the range $1.0-1.6$ $x 10^{6} \mathrm{~K}$, and with a low degree of ciruclar polarization.

Generally there are remarkably few structural changes in the active region sources from one day to the next, suggesting that structural evolution is relatively slow. We present contour maps at $1.5 \mathrm{GHz}$ for each of the four days in the Figure. However one sharp change from the 11 th to the 12 th can be seen in the region at about S20W10 on the 11 th. This region shows a highly polarized feature trailing the primary peak over the active region on the $11 \mathrm{th}$. By the $12 \mathrm{th}$ this location has developed into a strong eastward-pointing extension, in conjunction with the rapid emergence of new positive magnetic flux in a region of predominantly negative flux. Another interesting change occurs on the 13 th, when an ephemeral region appears as a strong compact source close to disk centre.

E. R. Priest and V. Krishan (eds.), Basic Plasma Processes on the Sun, 523-524. (C) 1990 IAU. Printed in the Netherlands. 
Two noise storms were present at $0.33 \mathrm{GHz}$ all week and were all highly polarized: the R-polarized storm in the north on all days is a single source, which however shows individual bursts shifting in position within the envelope of the storm. The noise storms in the south are more interesting. The maps from the 17th show them to be of opposite polarizations but well separated spatially, corresponding rather well to the simple underlying bipolar magnetc structure evident in the magnetogram. Clear evidence for the degree of divergence of magnetic field lines in the corona can be seen in the structure of this region. There is definite limb brightening present in the $.33 \mathrm{GHz}$ maps on all days.

This research was performed with support from NSF grant ATM-8717157, NASA grant NAGW-1541 and NASA contract NAG 5-969. The very Large Array is a facility of the National Radio Astronomy Observatory, which is operated by Associated Universities, Inc., under contract with the National Science Foundation.
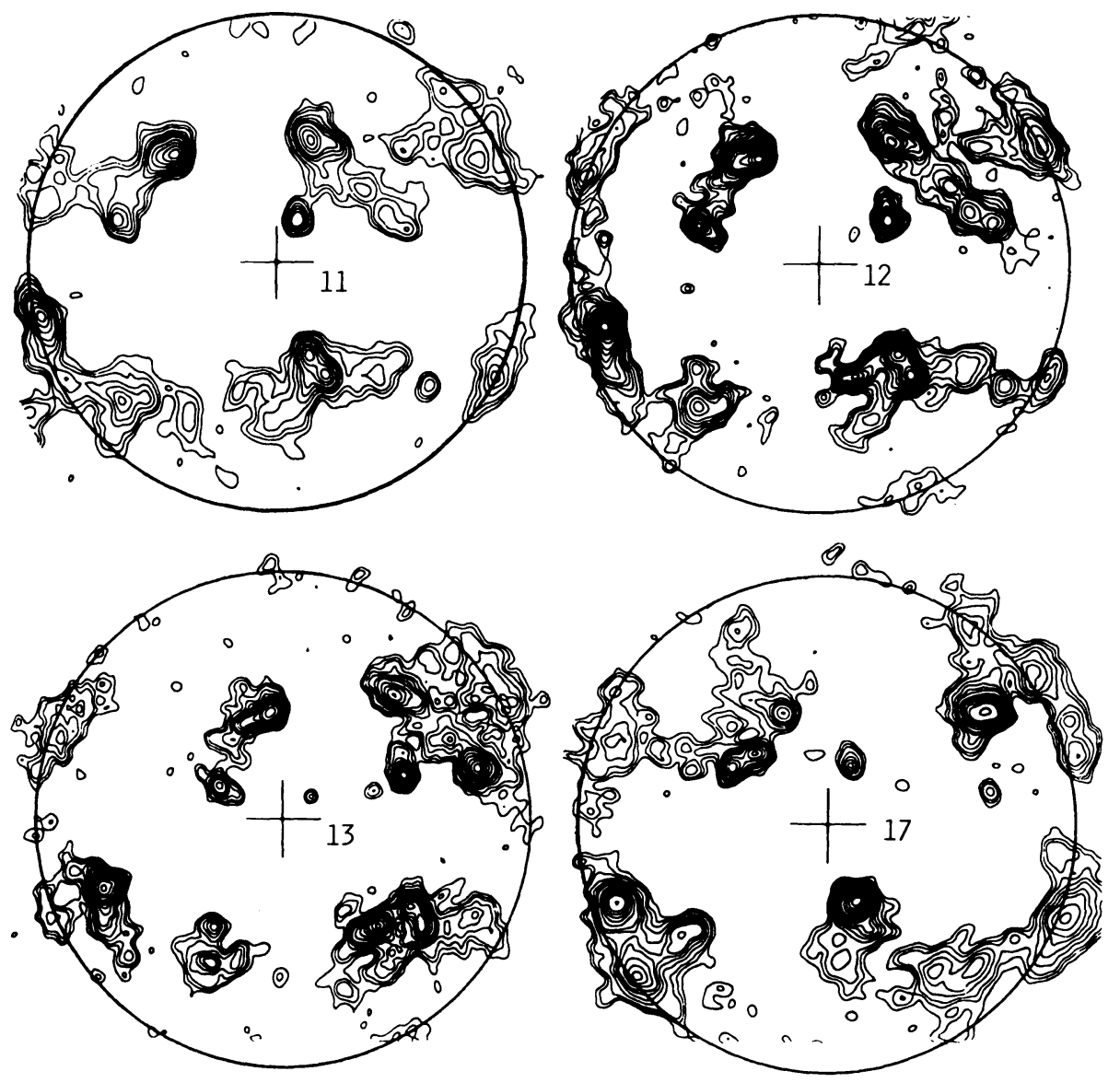